\title{
Updating of the Hierarchical Rock Mass Rating (HRMR) system and a new subsystem developed for weathered granite formations
}

\author{
Tiago Miranda ${ }^{1, *}$, L. Ribeiro e Sousa ${ }^{2}$, Joaquim Tinoco ${ }^{1}$ \\ ${ }^{1}$ ISISE, University of Minho, Guimarães, Portugal \\ ${ }^{2}$ State Key Laboratory of Geomechanics and Deep Underground Engineering, China University of Mining and Technology, Beijing, \\ China
}

\begin{abstract}
The RMR system is still very much applied in rock mechanics engineering context. It is based on the evaluation of six weights to obtain a final rating. To obtain the final rating a considerable amount of information is needed concerning the rock mass which can be difficult to obtain in some projects or project stages at least with accuracy. In 2007 an alternative classification scheme based on the RMR, the Hierarchical Rock Mass Rating (HRMR) was presented. The main feature of this system was the adaptation to the level of knowledge existent about the rock mass to obtain the classification of the rock mass since it followed a decision tree approach. However, the HRMR was only valid for hard rock granites with low fracturing degrees. In this work, the database was enlarged with approximately $40 \%$ more cases considering other types of granite rock masses including weathered granites and based on this increased database the system was updated. Granite formations existent in the north of Portugal including Porto city are predominantly granites. Some years ago a light rail infrastructure was built in the city of Porto and surrounding municipalities which involved considerable challenges due to the high heterogeneity levels of the granite formations and the difficulties involved in their geomechanical characterization. In this work it is intended to provide also a contribution to improve the characterization of these formations with special emphasis to the weathered horizons. A specific subsystem applicable to the weathered formations was developed. The results of the validation of these systems are presented and show acceptable performances in identifying the correct class using less information than with the RMR system.

Keywords: rock mass classification system, decision tree, weathered granite formations
\end{abstract}

\section{Introduction}

The RMR system ${ }^{[1]}$ allows the classification of the rock masses into five different classes related to their geotechnical properties. For each class it is possible to obtain support needs, type and excavation sequence, a range for the geomechanical parameters, stand-up time, among other information. For the classification process, the values of the six weights P1 to P6 - are needed in order to compute the RMR, which means that a great amount of geotechnical information has to be available and it can be difficult and/or expensive to obtain. All the classification process is deterministic, since the evaluation of the weight values to the final definition of the class. This can be a drawback since normally it is only possible to obtain approximate values of the weights or a possible range for them mainly in the preliminary stages of a project or when geological/geotechnical information is scarce. In this context, it was intended to develop an alternative classification scheme, based on the RMR that could overcome the mentioned difficulties.

The first version of the Hierarchical Rock Mass Rating (HRMR) was firstly presented by ${ }^{[2]}$ and it was developed using a database of 1222 cases of application of the RMR system in the scope of the construction of a large underground scheme called Venda Nova II. This scheme, built in the north of Portugal in a predominantly good quality granite rock mass, is almost fully composed by underground facilities, including caverns and several tunnels and shafts with total lengths of about $7.5 \mathrm{~km}$ and $750 \mathrm{~m}$, respectively ${ }^{[3,2]}$. To develop the HRMR a decision tree algorithm was applied to the database. Decision trees are branching structures based on split nodes, that test a given feature, and leaves, which assign a class label. This structure adapts very well to the objectives of this classification problem.

Hence, the HRMR is a classification system, with a decision tree configuration, which uses intervals for the weights of the RMR system to classify the rock mass. It is called hierarchical because it uses different levels of knowledge about the parameters and the classification accuracy depends on this knowledge level. The HRMR does not need the deterministic calculation of the RMR value to obtain a certain classification to the rock mass. It adapts to the level of knowledge about the parameters of the rock mass surpassing the deterministic definition of the classification weights used by this system and provides a probabilistically-based classification with a certain degree of accuracy. It was statistically validated using several performance measures. It is called hierarchical because it has four levels which provide a classification for the rock mass. Each level needs a different kind of knowledge about the rock mass, i.e. the deeper the knowledge the higher the classification accuracy.

The main limitations of the first version of the HRMR were related to the original database. In 
particular, this database was only composed by hard granite rocks with low fracturing degrees. In this work, the database was enlarged with 529 new cases (1751 total) and the HRMR system updated. The new cases are applications of the RMR system to granite rock masses gathered from different sources, namely the underground work of Bemposta II, also a hydroelectric scheme built in the North of Portugal in the international part of Douro river ${ }^{[4]}$, and the already cited case of Metro of Porto. This new version of the system considers a broader range of conditions of rock masses including some cases of weathered granites. Also a subsystem considering only the cases of weathered granite rocks from Metro of Porto was developed.

\section{Granite formations from metro of Porto}

The rock mass formations existing in the North of Portugal are predominantly granites. The most common minerals are quartz, feldspars and plagioclases, containing as major minerals micas (biotite and muscovite). These granite rock masses are characterized by high heterogeneity, which has significant implications in the design and characterization. Moreover, they have often very complex structures which provide a unique mechanical behavior and increased difficulties in the characterization process. In these granite formations there is a gradation from unaltered and sound rock to weathered rocks and stiff soils. Despite this typical gradation, frequently appear regions with stiff deformability materials completely separate from the global matrix.

Metro of Porto is a major light rail infrastructure built in the city of Porto and surrounding municipalities. In Porto's downtown, classified by UNESCO as World Heritage, the metro was built underground. The rock mass involved by metropolitan region of Porto city is in general a granite formation. A geomechanical classification was proposed for the granite formations adopting 7 groups, with the following design values presented at Tables 1 and $2^{[5]}$.

Table 1 Design geomechanical parameters for groups G1, G2, G3 and G4

\begin{tabular}{llllll}
\hline Geomechanical Groups & $\mathrm{UCS}(\mathrm{MPa})$ & $\gamma\left(\mathrm{kN} / \mathrm{m}^{3}\right)$ & $\mathrm{m}_{\mathrm{b}}$ & $\mathrm{s}$ & $\mathrm{E}(\mathrm{GPa})$ \\
\hline G1 & $90-150$ & $25-27$ & 7,45 & $6,9 \mathrm{E}-2$ & 35 \\
G2 & $30-90$ & $25-27$ & 3,2 & $7,5 \mathrm{E}-3$ & 10 \\
G3 & $10-35$ & $23-25$ & 0,98 & $7,5 \mathrm{E}-4$ & 1,5 \\
G4 & $1-15$ & $22-24$ & 0,67 & 0 & 0,5 \\
\hline
\end{tabular}

Table 2 Design geomechanical parameters for groups G5, G6, and G7

\begin{tabular}{lllll}
\hline Geomechanical Groups & $\gamma\left(\mathrm{kN} / \mathrm{m}^{3}\right)$ & $\mathrm{c}^{\prime}(\mathrm{MPa})$ & $\varphi^{\prime}\left(^{\circ}\right)$ & $\mathrm{E}(\mathrm{GPa})$ \\
\hline G5 & $19-21$ & $0,03-0,05$ & $34-36$ & 0,15 \\
G6 & $18-20$ & 0,01 & 32 & $0,03-0,05$ \\
G7 & $18-20$ & 0 & 28 & $0,02-0,03$ \\
G5 & $19-21$ & $0,03-0,05$ & $34-36$ & 0,15 \\
\hline
\end{tabular}

From a technical point of view, the most challenging problems were related with the unique heterogeneous characteristics of the granite rock mass due to weathering. The thickness of the weathered parts varies very quickly from several meters to zero. Weathered material, either transported or in situ, also occurs in discontinuities. A particularly striking feature is that, due to the erratic weathering of the granite, weathered zones of considerable size could be found under zones of sound granite ${ }^{[6]}$. A typical situation is Heroismo station where weathered granite with floating cores of granite occurs under a surficial part of a sound granitic rock mass (Fig. 1), the first underground station to be built. This station is located between the Campanhã and the Campo 24 de Agosto stations, which are inserted in the $\mathrm{C}$ line of Metro do Porto $^{[7]}$.

Another important station is the Bolhão station. In
Fig. 2 is presented a geological section that shows different geological groups distributed in depth since G2 (granite of excellent geomechanical quality) to G6 (residual soil granite). There is a zone of shallow landfill (G7) of small thickness and a large zone of G5, existing in these zone areas with stiff granite formations G2. It is apparent that the quality of the rock mass increases with depth, but beneath Fernandes Tomás street there is a probable fault zone consisting of material $\mathrm{G} 5^{[5]}$.

During construction of Porto Metro collapses occurred between 2000 and 2001. The project included two lines (Line $\mathrm{C}$ and $\mathrm{S}$ ) that included tunnels under the centre of the city. The tunnels were excavated by EPB shields and the heterogeneity was in the base of the accidents that occurred ${ }^{[8]}$. A typical section of weathered granite in the face of a TBM is illustrated in Fig. 3. 


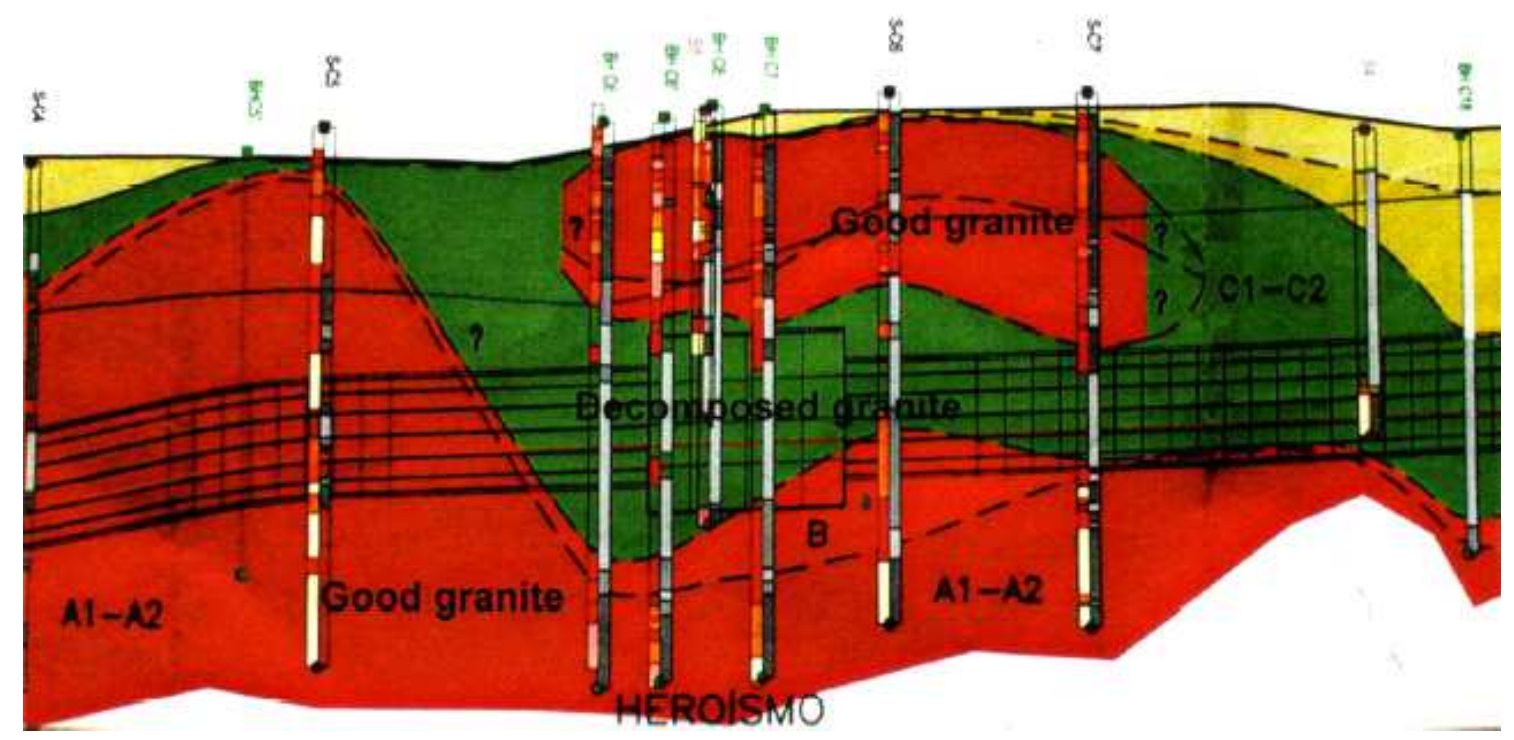

Fig. 1 Predicted geology for the Heroísmo station ${ }^{[6]}$

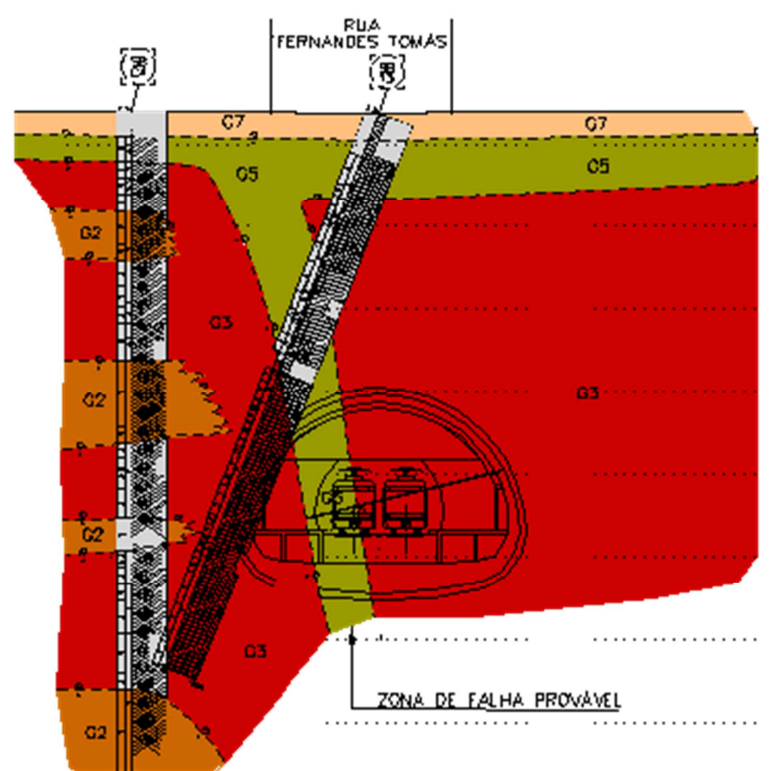

Fig. 2 Geological cross-section of Bolhão station ${ }^{[5]}$ 

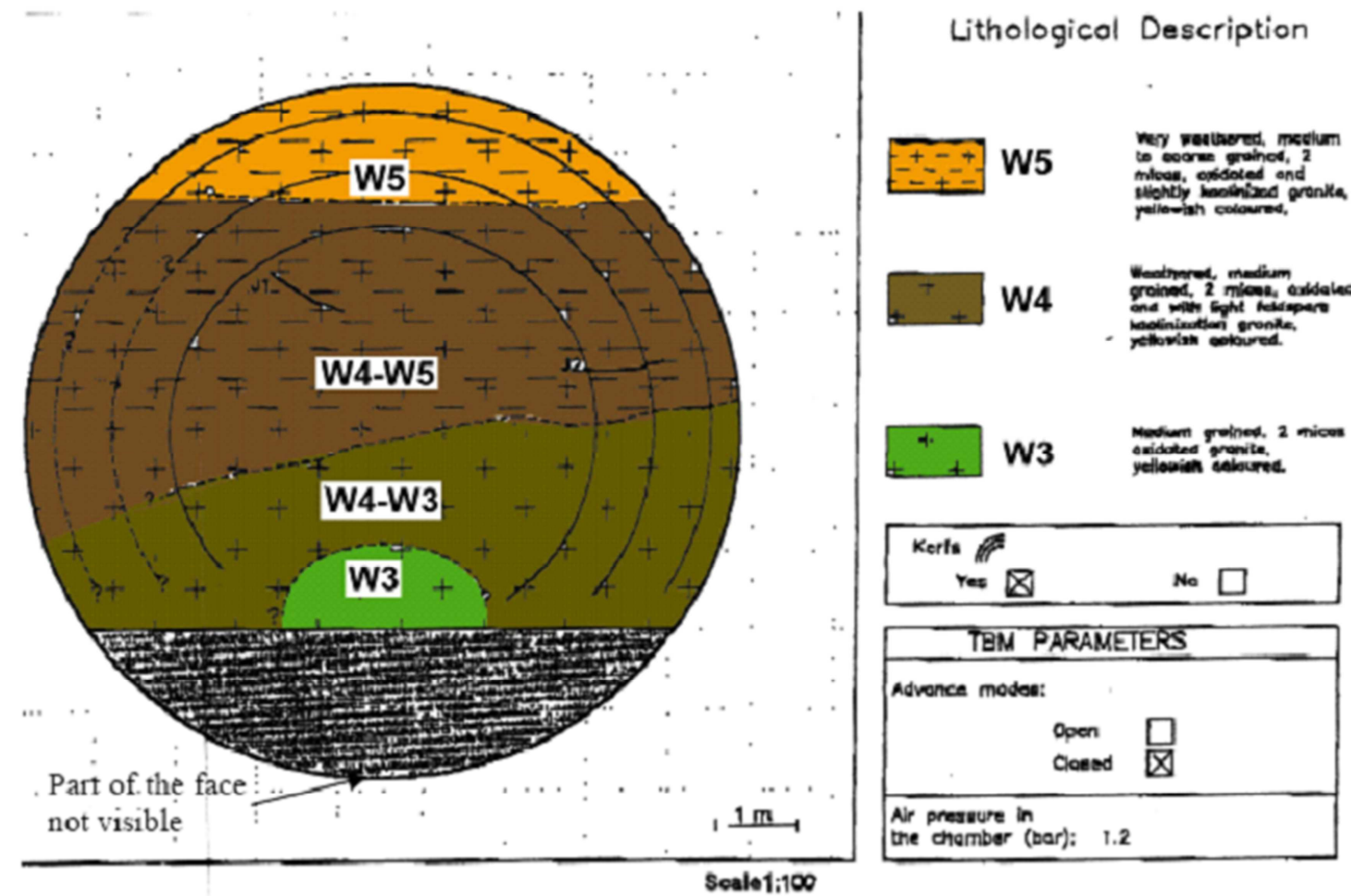

Fig. 3 As typical distribution of weathered granite in the face of the EPB tunnel ${ }^{[6]}$

\section{Data and models}

As stressed in the Introduction, in this work is an updating of the HRMR system is carried out aiming to develop a rock classification system with higher domain of applicability and also the development of a subsystem applicable to the weathered granites of Metro of Porto. Thus, based on new data meanwhile collected a new database with 1751 records was compiled covering soft and hard rocks, even though the later are still predominant.

The number of records of each class contemplated in this updated database is depicted in Fig. 4, where it is possible to observe that a significant number of records are classified as II and III (almost $85 \%$ of the records). On the other hand, for class $\mathrm{V}$ there are only 13 records, which certainly will influence the model performance in the identification of this class.

Fig. 5 shows the data distribution of the six RMR parameters in the database used in this work for model training and test. From its analysis, particularly based on P1 values (weight related with the unconfined compressive strength), it is possible to observe that the number of records related to medium/soft rocks is still limited but in the original HRMR they were almost totally absent. Even though high values of RQD are predominant (P2), in terms of joint spacing (P3) and conditions (P4) almost all range of values are covered. In terms of underground water (P5) slightly wet to dry conditions prevail and in terms of joint orientation (P6) a considerable broad band of situations are covered considering that most of the cases were gathered from underground works projects.

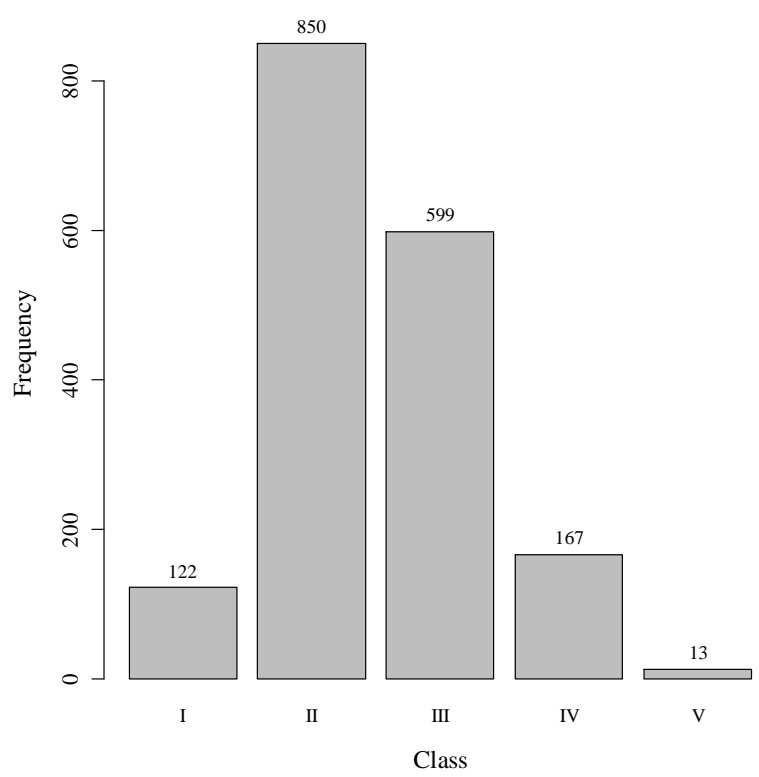

Fig. 4 Histogram of class frequencies in the database

Fig. 6 illustrates the correlation between all six parameters of RMR system, as well as its correlation with the RMR value. From its analysis, it is possible to conclude that the RMR has a strong correlation with P4. Moreover, P3 and P2 also present a good correlation with RMR. This facts point out to a good relation between the parameters related with joints and the overall rock mass condition. 

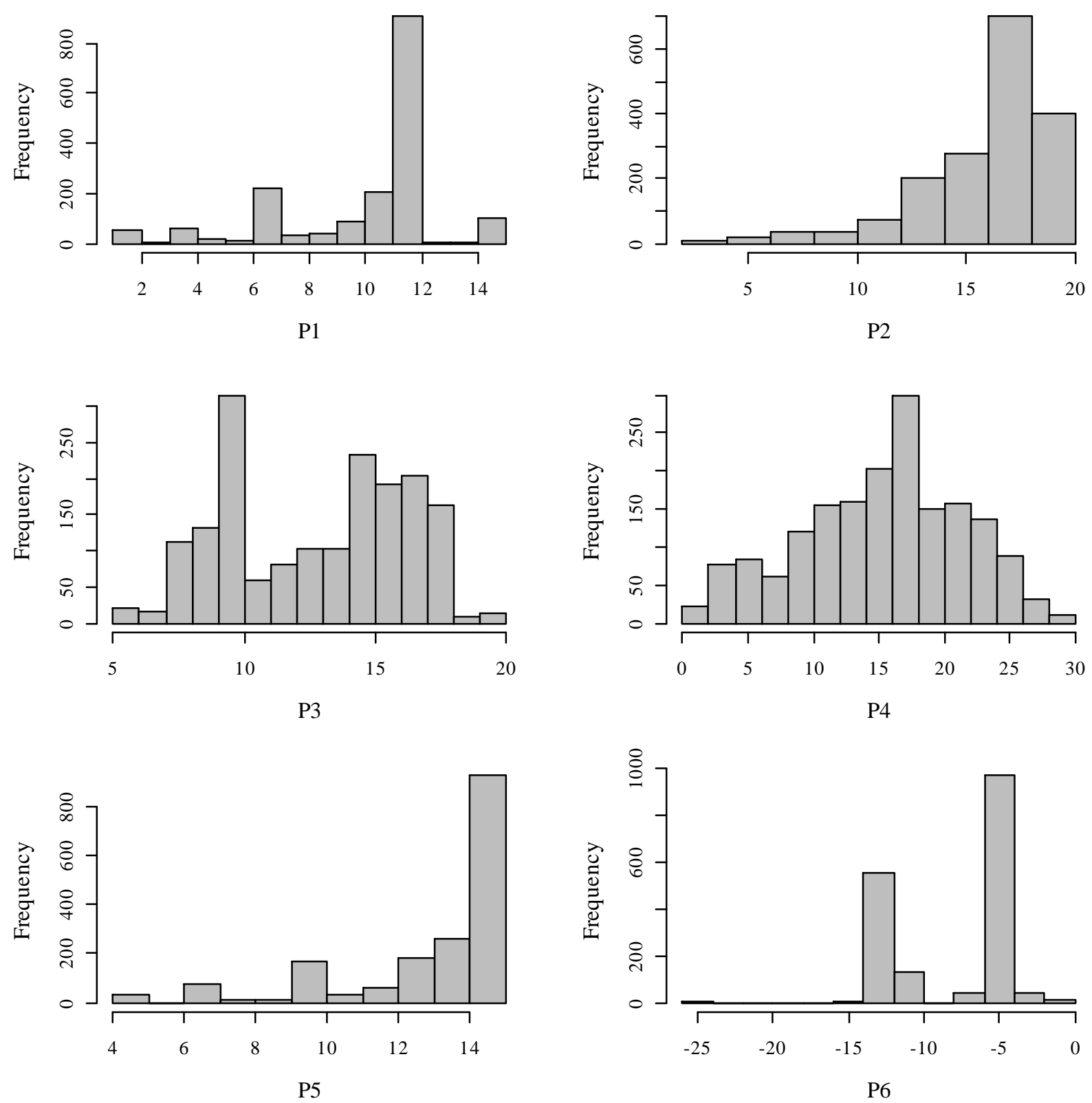

Fig. 5 Histogram of the six RMR parameters in the database 


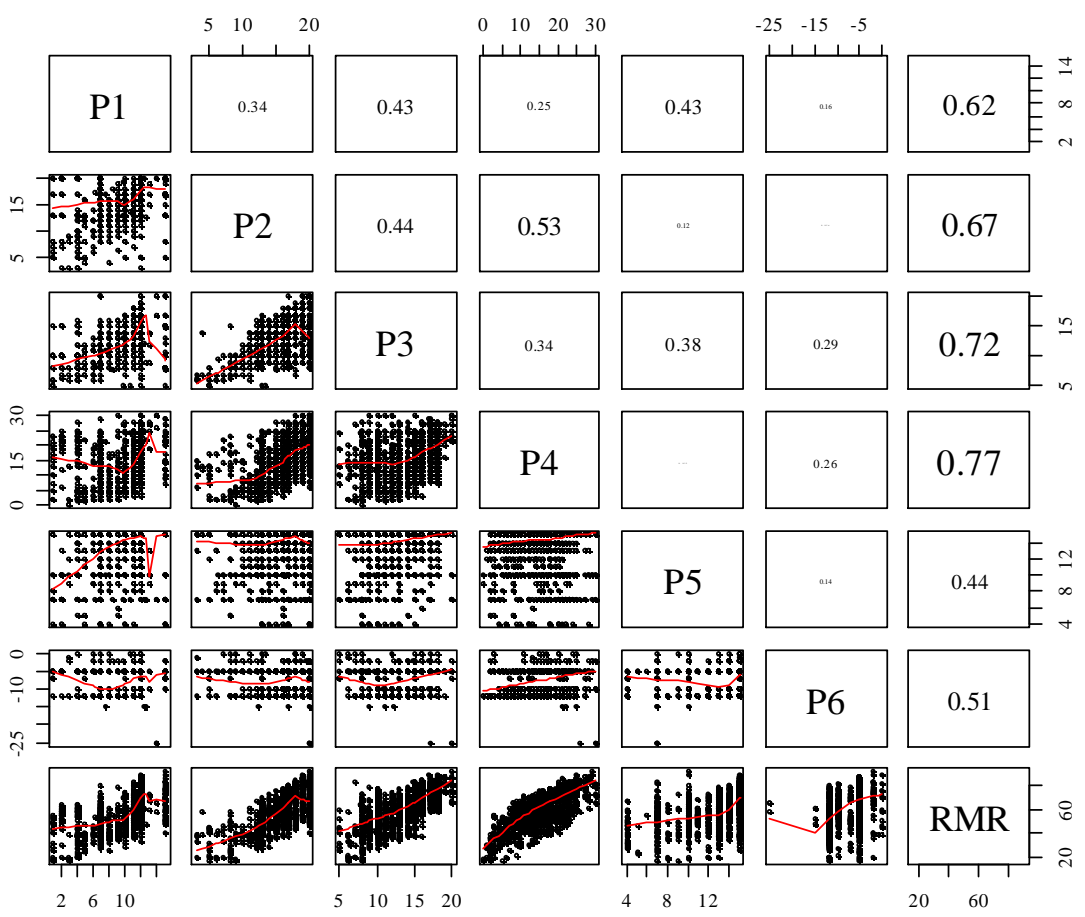

Fig. 6 Correlation matrix between all parameters in RMR system 
Concerning the algorithm used to develop the HRMR a decision tree is a direct and acyclic flow chart that represents a set of rules distinguishing classes or values in a hierarchical form. These rules are extracted from the data, using rule induction techniques, and appear in an "If-Then" structure expressing a simple and conditional logic. Source data is split into subsets, based on the attribute test value and the process is repeated in a recursive manner. Graphically they present a tree structure and are formed by three main components.

- The top node or root that represents all the data.

- Branches which connect nodes. Each internal node represents a test to an attribute while the branches denote the outcome of the test.

- Leafs which are terminal nodes represent classes or values.

After a tree is learned it can be used to classify or calculate the value of a new object. There are two types of decision trees, namely classification and regression trees ${ }^{[9]}$. These two types of trees use the same structure. The only difference is the type of the target variable. Classification trees are used to predict the class to which data belongs while regression trees are used to estimate the value of a continuous variable based on induced mathematical expressions.

The CART algorithm, the acronym for Classification And Regression Trees, developed by Breiman et al. $^{[10]}$, is one of the most popular algorithms used for inducing decision trees and was used in this work. It splits the data using a predictor that can be used several times at different levels. At each stage data is partitioned so that the cases of the two created subsets are more homogeneous than the previous one. It grows only binary trees (i.e., trees where only two branches can attach to a single root or node) so, even though its high flexibility, it can sometimes be unreliable and computationally slow.

CART algorithm is capable to construct trees which can be applied to analyze regression or classification problems with good results. Nevertheless, the fully automated process may result in an over structured inefficient tree. Moreover, many of the branches may reject noise or outliers in the training data. Tree pruning attempts to identify and remove such branches and simplify the tree, with the goal of improving accuracy on new data. The greatest benefits of decision trees approach are that they are easy to understand and interpret. They use a "white box" model, i.e. the induced rules are clear and easy to explain as they use a simple conditional logic. The main drawback is that they get harder to manage as the complexity of data increases leading to a higher number of branches in the tree.

The model generalization performance was accessed by 6 runs under a cross-validation $(k$-fold $=10)$ approach $^{[11]}$, where the data $(\mathrm{P})$ are randomly sampled into $k$ mutually exclusive subsets
$\left(P_{1}, P_{2}, \ldots, P_{k}\right)$, with the same length. Training and testing is performed $k$ times and the overall error of the model is taken as the average of the errors obtained in each iteration. Under this scheme, all of the data are used for training and testing. Yet, this method requires approximately $k$ (the number of subsets) times more computation, because $k$ models must be fitted. Based on the confusion matrix the overall accuracy was calculated. Moreover, for each level and for each class, the model sensibility was also determined. All experiments were implemented in the $\mathrm{R}$ tool ${ }^{[12]}$, using rminer library ${ }^{[13]}$.

\section{Results and discussion}

\subsection{Update of the HRMR system}

Fig. 8 shows the present version of the HRMR system. The decision tree is composed by four levels of classification. Each level provides the class of the rock mass with different accuracy degrees. The upper levels of the tree need less information but also have lower accuracy when compared with the lower levels.

The results of the classification are presented in the rectangular boxes in accordance to the RMR system (class I, II, ..., V). These boxes contain the class with higher probability to be correct. The decisions criteria are in the elliptical box (if true goes to the left side).

Table 4 shows the overall performance of the HRMR system based on the overall accuracy and sensitivity for each class and level. Sensitivity or recall is the percentage of cases that belong to a certain class that were classified as being of that particular class and accuracy is the percentage of correct predictions. These metrics range from 0 to $100 \%$, where low values indicate problems with the classifier and can be calculated as follows:

Table 3 Basis for accuracy and sensitivity calculation

\begin{tabular}{|c|c|c|}
\hline \multirow[b]{2}{*}{ Predicted } & \multicolumn{2}{|c|}{ Reference } \\
\hline & Event & No Event \\
\hline Event & A & B \\
\hline No Event & $\mathrm{C}$ & $\mathrm{D}$ \\
\hline
\end{tabular}

$$
\begin{gathered}
\text { Sensitivity }=A /(A+C) \\
\text { Accuracy }=(A+D) /(A+B+C+D)
\end{gathered}
$$

From Table 4 and following what was expected, the overall accuracy increases with the number of levels, i.e. as more specific knowledge about the weights is available. The highest increase is observed from Level 2 to Level 3 where the overall accuracy increases almost 6\%. However, if we perform a detailed analysis to the decision tree depicted in Fig. 8 , we can observe that the required information to apply Level 3 is the same for Level 4, i.e. all RMR parameters except P5. Therefore, we can conclude that Level 3 is redundant and thus the highest 
increase observed between two consecutives levels is from Level 2 to Level 4 (around 8\%). When compared to Level 1, Level 4 is around $12 \%$ more accurate. From Level 1 to Level 2 it is observed the lower accuracy increase (approximately 4\%). An overall assessment to the proposed HRMR system leads to the conclusion that Level 1 and Level 2 lacks of some accuracy and therefore should be used with caution. On the other side, Levels 3 and 4 present accuracy levels higher than $80 \%$ and this is possible using only an interval range for some of the weights.

Analyzing the results for each class, a performance increase is observed for every class with the number of levels. Class II and III are those with best performance. They have the highest values of sensitivity for almost all levels. This is closely related to the high number of cases classified as class II and III in the database, which represent almost $85 \%$ of the total number (Fig. 4). In contrast, class $\mathrm{V}$ has a very low number of cases (13). Therefore the algorithm has difficulties to learn its main features and the classification tree performs poorly for this class. In fact, sensitivity values are null for class $\mathrm{V}$ in every level. This means that the system is unable to classify as class $\mathrm{V}$ and should be used with caution for very poor rock masses. This may not be a decisive issue since very poor rock masses can be more easily classified as such in practice by experts than other classes.

Table 4 Performance measures for the HRMR system

\begin{tabular}{llll}
\hline & Class & Sensitivity $(\%)$ & Overall Accuracy $(\%)$ \\
\hline & I & 72.95 & \\
& II & 68.24 & \\
Level 1 & III & 80.47 & 72.82 \\
& IV & 74.25 & \\
& V & 0.00 & \\
\hline \multirow{5}{*}{ Level 2 } & I & 72.13 & \\
& II & 90.59 & 76.40 \\
& III & 62.10 & \\
& IV & 64.07 & \\
& V & 0.00 & \\
\hline \multirow{5}{*}{ Level 3 } & I & 72.13 & \\
& II & 90.24 & \\
& III & 80.13 & 82.35 \\
& IV & 64.07 & \\
& V & 0.00 & \\
\hline \multirow{2}{*}{ Level 4 } & I & 72.13 & \\
& II & 87.06 & \\
& III & 91.32 & 84.64 \\
& IV & 64.07 & \\
\hline
\end{tabular}

In a decision tree, the top nodes represent the most important data for classification. Fig. 7 shows the relative importance of each parameter in the HRMR system for classification matters (correspondent to level 4 which is more explanatory). It is interesting to observe that $\mathrm{P} 4$ is the most relevant parameter, although this parameter is not in the root node. The second most important parameter is $\mathrm{P} 3$ with an impact around $22 \%$. Both, $\mathrm{P} 3$ and $\mathrm{P} 4$ have an impact around $50 \%$. It is also interesting to observe that the three parameters most relevant are those considered by almost decisions nodes, i.e. P3, P4 and P6. This means that the parameters related with joints are the main predictors of the overall conditions of the rock mass.

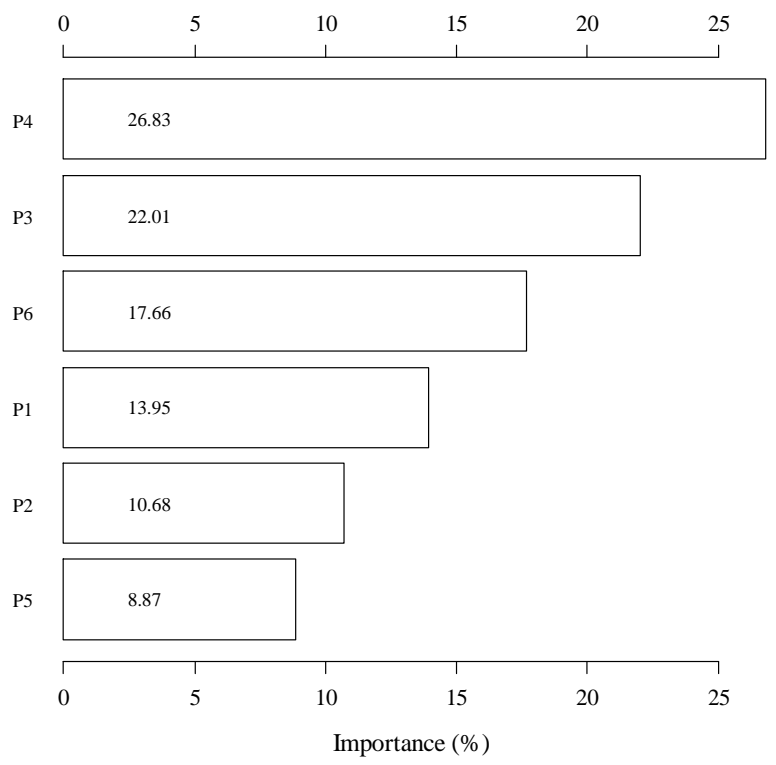

Fig. 7 Relative importance of the parameters in the HRMR system

\subsection{Specific subsystem for the weathered granite rock masses of Metro of Porto}

In order to develop a model specifically applicable for the weathered granites of Metro of Porto, only the records of correspondent to this project and with UCS $<20 \mathrm{MPa}$ were selected. In these conditions, a total of 118 records were used to train and test the model. In this set of examples there are no records classified as Class I.

Fig. 9 shows the HRMR system for these rocks. The decision tree is composed by two levels of classification. Each level provides the class of the rock mass with different accuracy degrees. The first level requires only P3 information but also have lower accuracy when compared with level 2.

Table 5 shows the overall performance of this subsystem, based on the overall accuracy and sensitivity for each class and level. From its analysis it can be concluded that when compared to level 1 , level 2 is around 10\% more accurate. Moreover, additionally to the information required by level 1 , level 2 requires only additional information conveyed by the P4 parameter, which eventually justifies an additional effort in obtaining such data. An overall assessment of the HRMR system proposed for these weathered rocks, lead us to underline that level 2 
present an interesting accuracy (higher than 84\%) and that level 1 should be applied with some caution.

Performing an analysis of the results by class, it is possible to observe that level 1 predicts very well classes III and IV, but is unable to classify correctly classes II and V. Using level 2, we achieved a very interesting accuracy for classes III and IV (87\% and 93\% respectively) and an average performance for class V (77\%). For class II, the classification tree was able to predict accurately just over half of the records $(55 \%)$. In both levels, class I cannot be predicted by the proposed HRMR system, which is closely related with the database used for model training as above underlined. However, since the proposed system is intended to be applied only to weathered granite rocks, the number of cases of class I rock masses should be low.
Table 5 Performance measures for the HRMR subsystem for the weathered rocks of Metro of Porto

\begin{tabular}{llll}
\hline & Class & Sensitivity $(\%)$ & Overall Accuracy $(\%)$ \\
\hline & I & NA & \\
\multirow{4}{*}{ Level 1 } & II & 0.00 & \\
& III & 90.74 & 75.42 \\
& IV & 100.00 & \\
& V & 0.00 & \\
\hline \multirow{4}{*}{ Level 2 } & I & NA & \\
& II & 54.55 & \\
& III & 87.04 & 84.75 \\
& IV & 92.50 & \\
& V & 76.92 & \\
\hline
\end{tabular}

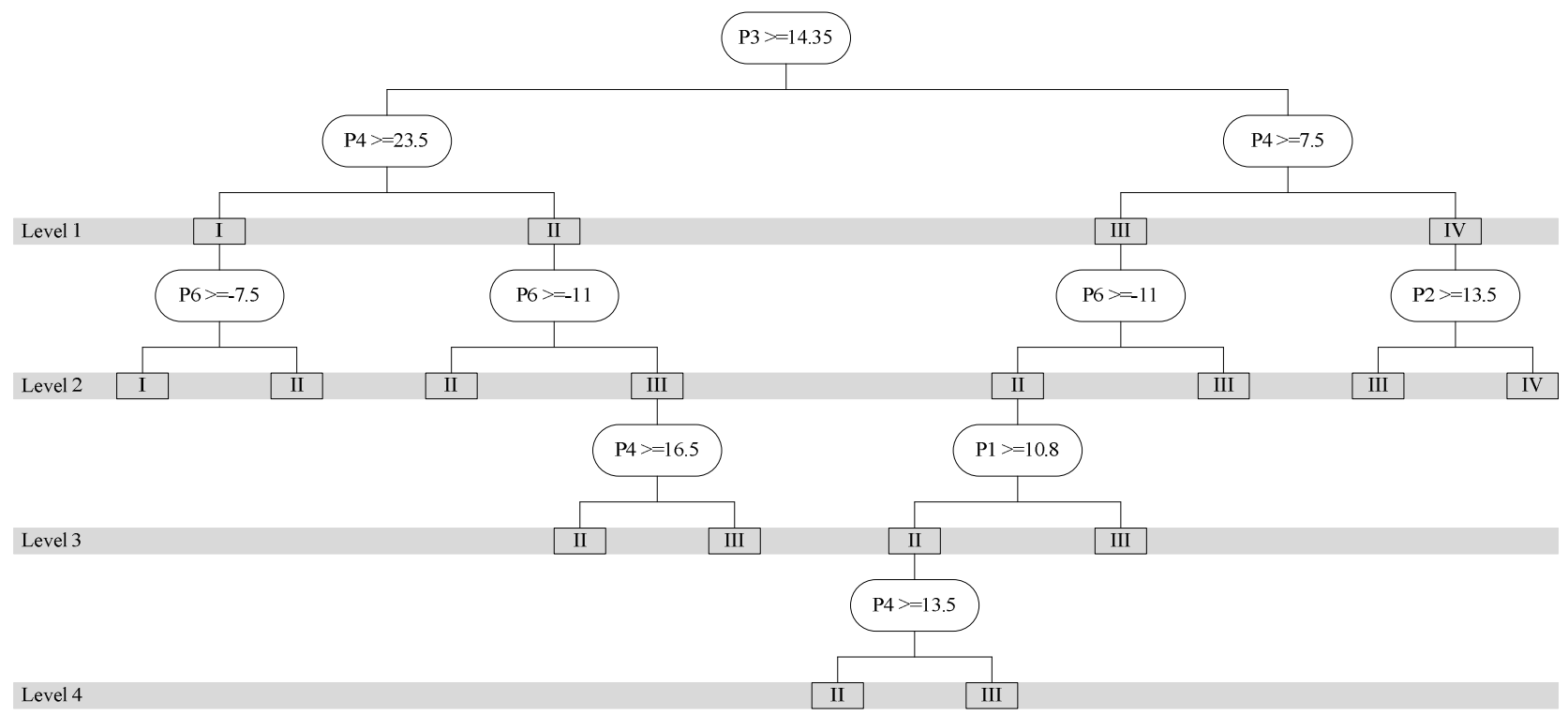

Fig. 8 The HRMR system

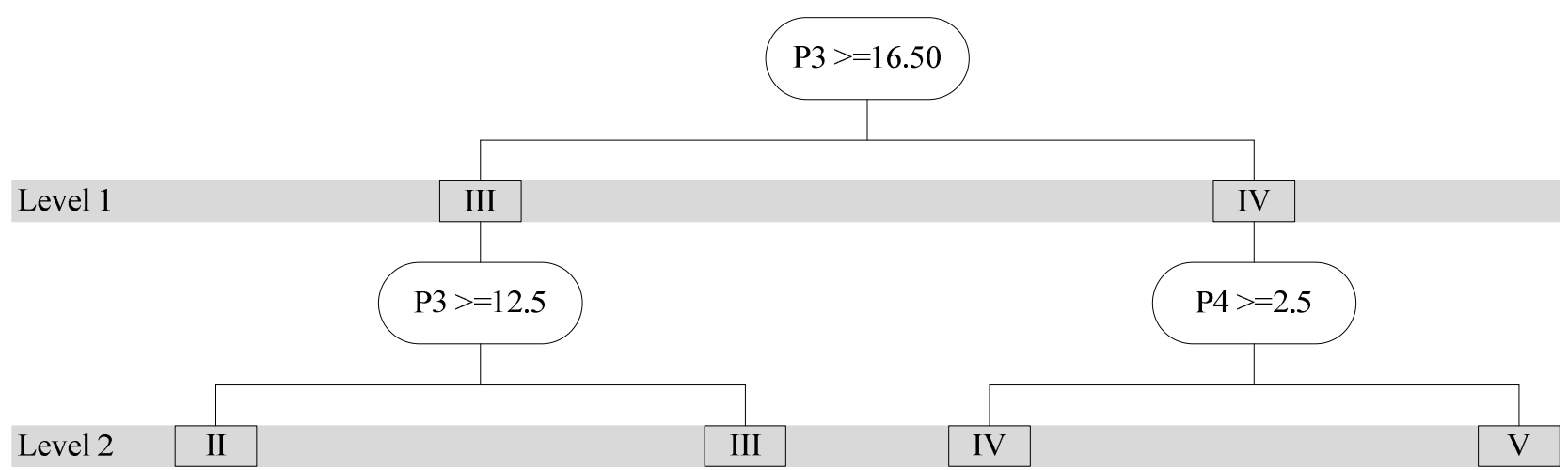

Fig. 9 HRMR subsystem for the weathered rocks of Metro of Porto 


\section{Final considerations}

In this work an update of the HRMR system was carried out by increasing the initial database in more than $40 \%$ of the cases. The initial system was applicable only for hard granite rocks and low fracturing degree. The present version considers a broader band of granite rock mass conditions. The system can be continuously improved as new data is gathered and the same approach can be adopted for other rock types.

This system tries to overcome some practical problems, namely in what concerns the difficulties to obtain some of the data needed for the RMR system application. As well as for other important classification systems, the RMR needs a precise definition of several parameters which involve the assembly of a considerable amount of geotechnical information. Some of this information can be difficult or expensive to obtain in the different design and construction stages.

The HRMR was developed by applying a decision tree algorithm. It is called hierarchical because it has four levels which provide a classification for the rock mass. Each level needs a different kind of knowledge about the rock mass, i.e. the deeper the knowledge the higher the classification accuracy.

The granite rock mass formations existent in the North of Portugal and in particular in Porto region are predominantly granites characterized by high levels of heterogeneity. In the Metro of Porto project this was a main feature and had a significant impact in geomechanical characterization, design and construction process. Therefore, it was intended in this work to contribute to the characterization of such formations by developing a specific tool that can be used in future projects.

The overall accuracy of the broader HRM is about $73 \%$ for level 1 whereas for level 4 it reaches almost $85 \%$. These results translate a very acceptable performance of the model in identifying the correct class with different levels of data. The results are better for Classes II and III due to the higher number of cases classified as such in the database. On the other hand the system is unable to classify class $\mathrm{V}$

\section{References}

[1] Bieniawski, Z.T. 1989. Engineering rock mass classifications. New York: Wiley.

[2] Miranda, T., 2007. Geomechanical parameters evaluation in underground structures. Artificial intelligence, Bayesian probabilities and inverse methods, $\mathrm{PhD}$ 's thesis. University of Minho, Guimarães, Portugal, 291 p.

[3] Lima, C., Resende, M., Plasencia, N. and Esteves, C. 2002. Venda Nova II hydroelectric scheme powerhouse rock masses. The most important parameters in the identification process are the ones related with joints (P3, P4 and P6) meaning that the parameters related with joints provide good indications concerning the overall quality of the rock mass.

The subsystem applicable to the weathered granites presents only two levels and level 2 presents $10 \%$ higher accuracy, approximately $84 \%$, than level 1 using only the additional information about parameter P4. Level 1 predicts very well classes III and IV, but is unable to classify correctly classes II and V. Level 2 provides a very interesting accuracy for classes III and IV (87\% and $93 \%$ respectively) an average performance for class $\mathrm{V}(77 \%)$ and a medium to poor performance for class II (55\%). In both levels, class I cannot be predicted due to limitations of the database.

The main characteristics of the HRMR are resumed in the following items.

- Does not need the deterministic definition of the weights of the RMR classification but only a range of values.

- Adaptation to the level of knowledge about the rock mass.

- Mainly uses data concerning the joints.

- It is based on a large number of cases and a solid statistical validation.

- Presents a good overall performance except in the prediction of poor rock mass conditions (class V).

In conclusion, the HRMR system can constitute as an interesting classification tool. It adapts to the level of knowledge about the rock mass providing a classification with different accuracy levels. It is based on a large database of cases and was properly validated in statistical terms. automatically, continuously and accurately, visualize the surface and underground structures vividly.

3) The system could calculate the location, area, coal column of coal pillars rapidly and accurately, which is valuable for calculating ore reserves and safeguarding the mining activity underground.

\section{Acknowledgements}

The authors would like to thank to EDP (Electricity of Portugal company) for making available part of the geotechnical information used in this work.

geotechnics and design. ISRM News Journal, 7(2): 37-41.

[4] Miranda, T.; Sousa, L.R. (2012). Application of Data Mining Techniques for the Development of Geomechanical Characterization Models for Rock Masses. In Innovative Numerical Modeling in Geomechanics, Ed. Sousa, Vargas, Fernandes \& Azevedo, Taylor \& Francis, London, p. 245-264.

[5] Miranda, T. 2003. Contribution to the calculation of geomechanical parameters for underground structures modelling in granite formations,. Master's thesis, 
University of Minho, Guimarães, Portugal (in Portuguese).

[6] Babendererde, S.; Hoek, H.; Marinos, P.; Cardoso, A.S. (2006). Geological risk in the use of TBMs in heterogeneous rock masses - The case of 'Metro do Porto', Geotechnical Risks in Rock Tunnels, Francis \& Taylor, Eds. Matos, Sousa, Kleberger \& Pinto, London, pp. 41-52.

[7] Ferreira, P.; Sousa, T.R.; Silva, P.; Vasconcelos, H.; Foged, N.; Sousa, L.R. (2007). Numerical Modeling and Monitoring Analysis of Heroísmo Station, Porto Metro. 11th ISRM Congress, Lisbon, p. 967-970.

[8] Sousa, R.L. (2010). Risk Analysis for Tunneling Projects. MIT, PhD Thesis, 599p.

[9] Berry, M. and Linoff, G. 2000. Mastering Data Mining: the art and science of customer relationships management. John Wiley \& Sons, Inc., USA.

[10] Breiman L., Friedman J. H., Olshen R. A., and Stone, C. J. 1984. Classification and Regression Trees. Wadsworth.

[11] Hastie, T., Tibshirani, R. and Friedman, J. (2009). The Elements of Statistical Learning: Data Mining, Inference, and Prediction. Springer-Verlag, New York, second edition.

[12] R Development Core Team, 2009 R: A language and environment for statistical computing. R Foundation for Statistical Computing, Vienna, Austria. ISBN 3-900051-00-3. Web site: http://www.r-project.org/.

[13] Cortez P., 2010. Data mining with neural networks and support vector machines using the R/rminer tool. In: Perner P. 10th Industrial conference on data mining, advances in data mining: applications and theoretical aspects. Berlin, Germany: LNAI 617: Springer, p. $572-583$. 
